\title{
Preliminary Study on the Healing Effect of Platelet-rich Fibrin for Soft Tissue Defects in Oral Implants
}

\author{
C. WANG* AND X. MA \\ Hebei Provincial Eye Hospital, Xingtai, Hebei 054001, China
}

Wang and Ma: Healing Effect of PRF for Soft Tissue Defects in Oral Implants

\begin{abstract}
Platelet-rich fibrin plays an active role in the repair of soft tissue defects in oral implants, but it still requires a long observation period, systematic description of platelet-rich fibrin to repair oral soft tissue defects in animal experiments to consolidate the relevant theoretical basis. New Zealand rabbits were selected as the test system. The experimental rabbits were randomly divided into 3 groups, platelet-rich fibrin group, collagen membrane group and blank control group. Each experimental rabbit was in the middle part of the hard palate, which was separated from the maxillary posterior incisors and left and right hard palate. A circular soft tissue full-thickness defect area was prepared by a tissue ring-cutting drill with a diameter of $5 \mathrm{~mm}$ at $2 \mathrm{~mm}$ of the mucosal edge. Three groups were implanted with platelet-rich fibrin membrane and collagen biofilm, and the blank control group was not treated. The healing morphology, wound healing rate, inflammatory response, collagen formation and angiogenesis were observed. The healing effect of soft tissue defects in platelet-rich fibrin oral implants was studied.
\end{abstract}

Key words: PRF, oral implant soft tissue defect, healing effect

In clinical implantation, factors affecting the long-term stability of an implant, in addition to osseointegration, as well as the healing of soft tissue around the implant, soft tissue deficiency and defects often affect the later aesthetic restoration ${ }^{[1]}$. Some commonly used soft tissue surgical methods, such as autologous connective tissue transplantation, free hernia transplantation, are difficult to master surgical methods ${ }^{[2]}$. Postoperative results are not good and also will add pain to patients ${ }^{[3]}$. At present, there are some disadvantages of commonly used allogeneic acellular dermal matrix and heterogeneous acellular dermal matrix, such as, ethical concerns, high price, risk of disease transmission, easy degradation, easy adhesion to bacteria, and increased chance of infection $^{[4]}$.

Platelet-rich fibrin (PRF) is a second-generation platelet concentrate that was reported by Hehn et al. ${ }^{[5]}$. The PRF contains all the components in the blood sample that are beneficial for healing and immunity ${ }^{[6]}$. It consists of polymerized fibrin and platelets, white blood cells, cytokines and circulating stem cells. It has a simple preparation, low cost and no need for anticoagulation ${ }^{[7]}$. The compressed PRF membrane has the advantages of elasticity and flexibility, and the cytokine can be stored for a long time and released slowly ${ }^{[8]}$. PRF is seen as a bio-material that promotes healing and opens the door to improved healing and regeneration in the oral cavity. In order to further explore the theoretical basis of PRF to promote the repair of oral soft tissue defects, this experiment established an animal experiment with a long observation period, observation of PRF to promote the healing of soft tissue defects by morphological and histological methods, that is the New Zealand rabbit hard sputum. The oral soft tissue full-thickness defect model was prepared. The autologous PRF membrane and the finished collagen membrane were implanted in the defect of the experimental group and the positive control group, respectively, and compared with the blank control group.

A total of 54 healthy male New Zealand white rabbits weighing 2.5-3.0 kg, were selected and caged for $1 \mathrm{w}$ under uniform conditions. Various treatments of experimental animals during the experiment met animal ethical requirements. The rabbits were randomly divided into 3 groups, the PRF group, the collagen membrane group, and the blank control group, with 18 rabbits in each group. In the middle part of the hard palate, soft tissue defects were prepared $2 \mathrm{~mm}$ from the incisors of the maxillary posterior incisors and the left and right 
hard palate mucosa. The mucoperior flap was removed to form a circular defect area, and the periosteal flap was removed from the edge of the defect. After adequate hemostasis, the wounds of the 3 groups were treated as follows, The PRF group had the autologous PRF membrane prepared before operation was sutured to the wound surface, and the membrane was in full contact with the wound base (bone surface); the collagen membrane group had the collagen biofilm was trimmed into the size of the wound was equivalent, and it was sutured and fixed at the wound ${ }^{[9]}$. The membrane was in full contact with the base of the wound (bone surface) and the blank control group had no treatment performed on the wound surface. This experiment aimed to compare the effects of PRF membrane and collagen biofilm in the repair of oral soft tissue defects, so it is necessary to prepare a certain size of soft tissue defects in the oral cavity of experimental animals. This study chose to prepare a soft tissue defect model in rabbit hard palate mucosa, and the soft tissue volume in this part is sufficient, which is convenient for surgical field exposure, surgical operation and dynamic observation of postoperative wounds. In this experiment, the 3 groups of rabbits were anesthetized on the $3 \mathrm{rd}$, 7th, 14th, 21st, 28th and 100th d after operation, and the oral cavity was exposed. The oral soft tissue defect healing of the experimental animals was observed by an oral reflector. Wound healing rate analysis was carried out. The area where the wound was not healed was calculated by transparencies and weighing method. The wound healing rate was calculated using the formula, wound healing rate $(\%)=$ (original wound surface cut-unhealed wound area)/original wound area) $\times 100$. Data were expressed as mean \pm standard deviation, and differences were considered statistically significant at $\mathrm{p}<0.05$.

Three days after surgery, the inflammatory response of the PRF group was mild, and the PRF membrane was firmly attached to the base of the wound in white. The collagen membrane group and the blank control group were red and swollen, and the bleeding was light; the collagen membrane was light yellow attached to the wound surface and the blank control group showed thin layer of fresh granulation tissue and a small amount of spotted hemorrhage. Seven days after surgery, most of the sutures of the 3 groups were selfshedding. The wound healing area of the PRF group was about $2 / 3$ of the original surface area and there was no obvious inflammatory reaction on the wound surface. In the other 2 groups, the inflammation of the wound improved, but the redness was still obvious.
Fourteen days after operation, the wound epithelium of the PRF group was completely covered, no tissue depression, no scar formation, and the color, shape and texture of the mucosa in the operation area were completely consistent with the normal mucosa ${ }^{[10]}$. The wound healing tissue of the collagen membrane group and the blank control group was in a concave state, but the basement had a mucosal epithelium covering, and the latter had a larger extent of depression. Twentyone days after surgery, the collagen membrane group healed the wound without tissue depression, but there was scar formation. The blank control group still had a certain degree of tissue depression and scars were visible. Twenty eight days after surgery, the wounds in the blank control group healed completely and there was no tissue depression. Fifty six days after operation, the morphology of the PRF group recovered well without scars; the collagen membrane group and the blank control group recovered poorly and the scar was obvious. The area of the wound in the oral cavity of all experimental animals was gradually reduced over time. At the six time points set, it was observed that the soft tissue defects of PRF group, collagen membrane group and blank control group were completely healed at $14 \mathrm{~d}, 21 \mathrm{~d}$ and $28 \mathrm{~d}$ after operation and no tissue depression and it's wound healing rate was $100 \%$. Therefore, the wound healing rates only compared at 3 and $7 \mathrm{~d}$ after surgery. The results of the analysis showed that there was no significant difference in the wound healing rate among the different experimental groups $3 \mathrm{~d}$ after surgery $(\mathrm{p}>0.05)$. Seven days postoperatively, the wound healing rate between the PRF group and the collagen membrane group was statistically significant $(p<0.05)$. The difference between the wound healing rate of the collagen membrane group and the PRF group was statistically significant. The wound healing rate $7 \mathrm{~d}$ after surgery is a $\mathrm{s}$ follows, PRF group $>$ collagen membrane group $>$ blank control group (Table 1).

Three rabbits were randomly sacrificed 3, 7, 14, 21, 28 and $56 \mathrm{~d}$ after surgery with $3 \%$ pentobarbital sodium. The normal tissue part of the hard sputum area and the $2 \mathrm{~mm}$ area around the operation area was cut out, and hematoxylin and eosin (HE) stained sections were taken. The HE stained sections were observed under a 40-fold, 100-fold and 400-fold light microscope using a digital trinocular camera microscopic camera system. The number of inflammatory cells in the tissues was counted under the microscope and pathologically graded. The specific scoring criteria are as follows, 3 and $7 \mathrm{~d}$ after surgery, the inflammatory conditions of the 3 groups were different $(p<0.05)$. The results of the 
TABLE 1: WOUND HEALING RATE IN 3 GROUPS OF RABBITS AT DIFFERENT TIMES AFTER INJURY

\begin{tabular}{lcc}
\hline Experimental grouping & 3 days & $\mathbf{7}$ days \\
\hline PRF group & $14.31 \pm 2.15$ & $66.90 \pm 8.47^{\star \wedge}$ \\
Collagen membrane group & $10.22 \pm 2.98$ & $44.52 \pm 2.47^{*}$ \\
Blank control group & $6.17 \pm 4.76$ & $30.35 \pm 2.22$ \\
\hline
\end{tabular}

${ }^{*} \mathrm{p}<0.05$ compared to the blank group control; ${ }^{\wedge} \mathrm{p}<0.05$ compared with the collagen membrane group. Values are mean \pm standard deviation of $\%$ wound healing of $n=3$ observations

pairwise comparison between the PRF group and the other 2 groups were statistically significant $(p<0.05)$; there was no difference between the collagen membrane group and the blank control group, which was not statistically significant $(\mathrm{p}>0.05)$. The inflammatory response of the PRF group was smaller than that of the collagen membrane group and the blank control group at 3 and $7 \mathrm{~d}$ after surgery. There were no significant differences in inflammation between the three groups at $14,21,28$, and $56 \mathrm{~d}$ after surgery $(\mathrm{p}>0.05)$. It is indicated that after $7 \mathrm{~d}$, there was no difference in the inflammatory response of the three groups.

Three, 28, and 56 days after surgery, there was no significant difference in the mean optical density of CD31 between the experimental groups, which was not statistically significant $(1>>0.05)$. Seven and $14 \mathrm{~d}$ after operation, the mean optical density of CD31 in PRF group was higher than that in collagen membrane group and blank control group, the difference was statistically significant $(\mathrm{p}<0.05)$ and the average optical density of CD31 in collagen membrane group was higher than blank. In the control group, the difference was statistically significant $(\mathrm{p}<0.05)$. At $21 \mathrm{~d}$ after operation, the mean optical density of CD31 in the PRF group was higher than that in the collagen membrane group and the blank control group, the difference was statistically significant $(\mathrm{p}<0.05)$. There was no significant difference in the average optical density of CD31 between the collagen membrane group and the blank control group ( $\mathrm{p}>0.05)$.

Three days after surgery, there was no significant difference in the mean optical density of collagen fibers in each experimental group ( $p>0.05)$. Seven days after operation, the mean optical density of collagen fibers in the PRF group was significantly lower than that in the collagen membrane group and the blank control group $(p<0.05)$. The collagen membrane group had higher average optical density than the blank control group. The difference was statistically significant $(\mathrm{p}<0.05)$. Fourteen days after operation, the mean optical density of collagen fibers in PRF group and collagen membrane group was higher than that in the blank control group, and the difference was statistically significant $(\mathrm{p}<0.05)$. There was no significant difference in the mean optical density of collagen fibers between the PRF group and the collagen membrane group. Twenty one, 28 and $56 \mathrm{~d}$ after operation, the mean optical density of collagen fibers in the PRF group and the collagen membrane group was lower than that in the blank control group, the difference was statistically significant $(p<0.05)$; average light of collagen fibers in the PRF group the density value was lower than that of the collagen membrane group, and the difference was statistically significant $(\mathrm{p}<0.05)$.

Once the wound is formed, the first reaction of the body is to stop bleeding, and then the exposed collagen fibers direct the platelets to aggregate to form a blood clot, which in turn is the source of autologous growth factors. Blood clots are the center of soft tissue healing and its formation marks the beginning of the healing process. Studies have confirmed that PRF can provide a more stable fibrin network scaffold than blood clots, and it plays a decisive role in tissue repair in the three major components of PRF. It is like a cell scaffold that can effectively direct the migration of stem cells to the wound, especially to induce epithelial cells to migrate to the wound surface and accelerate the healing process. In addition, PRF fibrin regulates the processes associated with wound healing processes by combining various growth factors, such as initial angiogenesis, epithelial coverage, and immune regulation. It also regulates the metabolism of fibroblasts and the accelerates of the healing of soft tissues. Studies have shown that the concentration of platelets in PRF is 3-7 times higher than normal blood. The $\alpha$-granules in platelets can release a variety of growth factors that promote wound healing, such as epidermal growth, platelet-derived growth, vascular endothelial growth, which not only have synergistic effects, but other cell-promoting activities. Factors interact with each other, making soft tissue wounds heal faster and better. In addition, in the PRF group, no soft tissue was found to have a concave healing during the healing process, and it was observed that this phenomenon also appeared in the other two groups. At the same time, it was observed that the soft tissue defects of the PRF group, the collagen membrane group and the blank control group were completely healed at the 14, 21 and $28 \mathrm{~d}$ after surgery, and the wound tissue was finally healed. In the PRF group, no obvious scars were observed, while the other two groups formed obvious scars visible to the naked eye. It shows that PRF can reduce the formation of scars and improve the quality of wound healing. Combined with 
morphological observations, it is further demonstrated that PRF can accelerate the repair process of soft tissue defects.

Inflammation is the basic defense response against external infections and injuries after trauma, and it is also the first stage of post-mechanical healing. PRF has been shown to control the inflammatory response and has a supporting effect on the immune system, which can reduce the incidence of postoperative infection. In this study, the soft tissue inflammatory cell infiltration in the wound area was observed by HE staining and the pathological grading score was performed to evaluate the inflammatory response of the three groups of wounds at various time points after operation (Table 2). At 3 and $7 \mathrm{~d}$ after surgery, the inflammatory conditions of the 3 groups were different $(p<0.05)$. The results of the pairwise comparison between the PRF group and the other 2 groups were statistically significant $(\mathrm{p}<0.05)$; There was no difference between the collagen membrane group and the blank control group. It could be noted that the inflammatory response of the PRF group was lower than that of the other 3 groups 3 and $7 \mathrm{~d}$ after surgery, which was consistent with the morphologically observed results. Fourteen, 21, 28 and $56 \mathrm{~d}$ after surgery, there was no significant difference in inflammation between the 3 groups. It is indicated that after $7 \mathrm{~d}$, the inflammatory reactions of the 3 groups have not changed. There was no obvious inflammatory reaction in the PRF group, no bleeding was detected. The degree of inflammatory cell infiltration was lower in HE staining than in the other 2 groups, further indicating that PRF can effectively reduce the incidence of inflammation and infection

TABLE 2: PATHOLOGICAL INFLAMMATION GRADING AND SCORING

\begin{tabular}{lcc}
\hline Grading & Mirror description & Score \\
\hline- & $\begin{array}{c}\text { Basically normal, inflammatory cells } \\
\text { are occasionally present or absent } \\
\text { Scattered infiltration of a few }\end{array}$ & 0 \\
++ & $\begin{array}{c}\text { numbers of inflammatory cells } \\
\text { Between }+,++\end{array}$ & 1 \\
++ & $\begin{array}{c}\text { A large number of diffuse } \\
\text { inflammatory cells infiltrate, showing } \\
\text { multinucleated giant cells forming } \\
\text { inflammatory granulomatous lesions }\end{array}$ & 3 \\
\hline
\end{tabular}

during wound healing. Especially in the week after trauma, its antiinflammatory effect is significant, which has a positive impact on wound healing.

Studies have shown that wound neovascularization is the main determinant of wound healing. In the middle and late stages of wound healing, the amount of new blood vessels in the PRF group was still higher than the other two groups, which played a positive role in the further remodeling of the wound. The reason for the above results is that PRF is a natural fibrin biomaterial that is beneficial to the formation of micro-vessels. The three-dimensional network structure of fibrin conforms to the physiological structure and helps guide the migration of endothelial cells. The structure has a good elasticity and large pores, which is beneficial to the diffusion of oxygen and nutrients and participate in the process of vascularization. In addition, the fibrin matrix itself does have angiogenic properties. Studies have shown that the release of growth factors in PRF lasts at least $1 \mathrm{w}$ and peaks at $7-14 \mathrm{~d}$ in $28 \mathrm{~d}$. Stimulated by these angiogenic growth factors, a.vp.3 integrin, which is expressed at a low level on the surface of the endothelial cells at rest, is up-regulated, which not only binds vascular endothelial cells to fibrin, fibronectin and the vitronectin can also regulate the proliferation and apoptosis of endothelial cells, thereby playing an active role in angiogenesis. As the wound gradually healed, the amount of PRF released growth factor decreased gradually, the destruction and consumption of wound growth factors and the gradual disintegration of fibrin scaffolds caused the wound angiogenesis to weaken. There is no significant difference in neovascularization in the three groups $21 \mathrm{~d}$ after surgery (Table 3 ).

Fibroblasts are one of the main repair cells of wounds. Their main function is to synthesize collagen fibers, which play an important role in the whole wound healing process. In the early stage of wound repair, the synthesis of collagen fibers is much more decomposition, and the arrangement is more disordered. At the shaping stage, the decomposition is more than synthesis, and the arrangement is gradually regular. If the degradation is hindered, the wound will be excessively fibrillated to form scars. In the analysis of the average optical density values of collagen fibers 21,28 and $56 \mathrm{~d}$ after operation,

TABLE 3: COMPARISON OF WOUND INFLAMMATION SCORES IN THE 3 GROUPS OF RABBITS AT DIFFERENT TIME POINTS AFTER INJURY

\begin{tabular}{lcccccc}
\hline Grouping & $\mathbf{3}$ days & $\mathbf{7}$ days & 14 days & $\mathbf{2 1}$ days & $\mathbf{2 8}$ days & 56 days \\
\hline PRF group & $1.67 \pm 0.58^{\star \wedge}$ & $1.00 \pm 0.00^{\star \wedge}$ & $1.00 \pm 0.00$ & $0.67 \pm 0.58$ & $0.67 \pm 0.58$ & $0.33 \pm 0.58$ \\
Collagen membrane group & $3.00 \pm 0.00$ & $2.33 \pm 0.58$ & $1.67 \pm 0.58$ & $1.33 \pm 0.58$ & $1.00 \pm 0.00$ & $0.33 \pm 0.58$ \\
Blank control group & $3.00 \pm 0.00$ & $2.67 \pm 0.58$ & $2.00 \pm 0.00$ & $1.67 \pm 0.58$ & $1.33 \pm 0.58$ & $0.33 \pm 0.58$ \\
\hline
\end{tabular}

${ }^{*} \mathrm{p}<0.05$ compared to the blank group control; ${ }^{\wedge} \mathrm{p}<0.05$ compared to the collagen membrane group 
TABLE 4: COMPARISON OF OPTICAL DENSITY OF WOUNDS AND COLLAGEN FIBERS IN THE 3 GROUPS OF RABBITS AT DIFFERENT TIME POINTS AFTER INJURY

\begin{tabular}{|c|c|c|c|c|c|c|}
\hline rouping & 3 days & 7 days & 14 days & 21 days & 28 days & 56 days \\
\hline \multicolumn{7}{|c|}{ Comparison of average optical density values of wounds } \\
\hline PRF group & $0.2655 \pm 0.0083$ & $0.3235 \pm 0.0095^{* \wedge}$ & $0.3184 \pm 0.0018$ & $0.2992 \pm 0.0030$ & 0.286 & 0.27 \\
\hline $\begin{array}{l}\text { Collagen } \\
\text { membrane group }\end{array}$ & $0.2566 \pm 0.0059$ & $0.2890 \pm 0.0014^{*}$ & $0.2906 \pm 0.0009$ & $0.2800 \pm 0.0086$ & 141 & 109 \\
\hline Blank control group & $2624 \pm 0.0086$ & $0.2737 \pm 0.0005$ & $0.2780 \pm 0.0013$ & 0.2790 & 0.268 & $0.2624 \pm 0.0052$ \\
\hline \multicolumn{7}{|c|}{ Comparison of average optical density values of collagen fibers } \\
\hline up & $0.3792 \pm 0.0097$ & $0.4496 \pm 0.0114$ & $0.4362 \pm 0.0052$ & $0.4092 \pm 0.0030$ & $0.3095 \pm 0.0098$ & 0.387 \\
\hline $\begin{array}{l}\text { ollagen } \\
\text { nembrane group }\end{array}$ & 62 & $0.4098 \pm 0.0032$ & 7 & 0. & 6 & 009 \\
\hline Blank control group & $0.3669 \pm 0.0048$ & $0.3817 \pm 0.0042$ & $0.4224 \pm 0.0011$ & $0.4383 \pm 0.0013$ & $0.4292 \pm 0.0048$ & $0.4198 \pm 0.0079$ \\
\hline
\end{tabular}

${ }^{*} \mathrm{p}<0.05$ compared to the blank group control; ${ }^{\wedge} \mathrm{p}<0.05$ compared to the collagen membrane group. Optical density values are expressed as mean \pm standard deviation of $\mathrm{n}=3$ observations

the average optical density values of collagen fibers in the PRF group and the collagen membrane group were lower than those in the blank control group, and the difference was statistically significant $(\mathrm{p}<0.05)$; compared with the collagen membrane group, the average optical density of collagen fibers in the PRF group was lower, and the difference was statistically significant $(p<0.05)$. In the middle and late stages of wound healing, the collagen content in the PRF group $<$ collagen membrane group $<$ blank control group, and the collagen fibers in the collagen membrane group and the blank control group were deeply stained, and the arrangement was relatively disordered. In combination with morphological observation, in the healing process, scar formation was observed in the collagen membrane group and the blank control group, but no scar was found in the wound of the PRF group. When the wound was completely healed, the scar of the collagen membrane group and the model control group were obvious. Due to scar traction, the recovery of wound morphology is not ideal, but the wound surface of PRF group is not obvious. Moreover, the morphology of wound is no different from normal tissue, so it showed that PRF can reduce scar formation. In addition, growth factors can regulate collagen degradation, so we speculate that PRF may also balance the synthesis and degradation of collagen through its rich growth factors, thereby reducing scar formation (Table 4).

PRF can promote the repair of oral soft tissue defects more quickly, and there is no tendency for soft tissue to heal. PRF can reduce the inflammatory reaction during wound healing, especially in the early stage of trauma (within $1 \mathrm{w}$ after trauma). It is obvious that PRF can accelerate the process of neovascularization of wounds and promote early healing of wounds; PRF can promote collagen deposition in wounds in the early stage of trauma, accelerate wound healing, regulate collagen metabolism in the middle and late stages of trauma, reduce scar formation and improve wound healing quality. In short, PRF is endogenous and has considerable biosafety. It is an activation platform for promoting oral soft tissue regeneration, which can effectively regulate the whole process of wound healing according to the physiological laws of wound healing. This experiment closely follows the practical problems of clinical soft tissue repair and regeneration in oral implants, and comprehensively explores the advantages of PRF to promote soft tissue regeneration, which has obvious clinical significance. This investigation established an animal model with a long observation period (56 d), which showed that the PRF to promote oral soft tissue defects through morphological and histological systems, further enriching the basic theoretical content of the research field. The shortcomings and extensions of this topic: there is no unified conclusion on how PRF regulates scar formation, and further research is needed.

\section{REFERENCES}

1. Boora P, Rathee M, Bhoria M. Effect of Platelet Rich Fibrin (PRF) on Peri-implant Soft Tissue and Crestal Bone in OneStage Implant Placement: A Randomized Controlled Trial. J Clin Diagn Res 2015;9(4):18-21.

2. Öncü E, Alaaddinoğlu EE. The effect of platelet-rich fibrin on implant stability. Int $\mathrm{J}$ Oral Maxillofac Implants 2015;30(3):578.

3. Gaži P, Pietrzak K, Tessaro S. The Exact PRF Security of Truncation: Tight Bounds for Keyed Sponges and Truncated CBC. In: Gennaro R, Robshaw M, editors. Advances in Cryptology- CRYPTO 2015. Lecture Notes in Computer Science. Volume 9215. Berlin, Heidelberg: Springer; 2015. p. 368-87.

4. Hiremath H, Saikalyan S, Kulkarni SS, Hiremath V. Secondgeneration platelet concentrate (PRF) as a pulpotomy medicament in a permanent molar with pulpitis: a case report. Int Endod J 2015;45(1):105-12.

5. Hehn J, Schwenk T, Striegel M, Schlee M. The effect of PRF (platelet-rich fibrin) inserted with a split-flap technique on soft tissue thickening and initial marginal bone loss around 
implants: results of a randomized, controlled clinical trial. Int J Implant Dent 2016;2:13.

6. Kobayashi E, Flückiger L, Fujiokakobayashi M, Sawada K, Sculean A, Schaller B, et al. Comparative release of growth factors from PRP, PRF, and advanced-PRF. Clin Oral Investig 2016;20(9):2353-60.

7. Kumar PG, Kumar GS, Kumar SCV. Platelet Rich Fibrin (PRF) in Regeneration of Intrabony Defects- A Randomized Controlled Trial. J Periodontol 2017;88(11):1-14.

8. List E, Nandi M. Revisiting Full-PRF-Secure PMAC and Using It for Beyond-Birthday Authenticated Encryption. In: Handschuh H, editor. Topics in Cryptology - CT-RSA 2017. CT-RSA 2017. Lecture Notes in Computer Science. Volume 10159. Cham, Switzerland: Springer; 2017. p. 258-74.

9. Marenzi G, Riccitiello F, Tia M, Di LA, Sammartino G.
Influence of Leukocyte- and Platelet-Rich Fibrin (L-PRF) in the Healing of Simple Postextraction Sockets: A Split-Mouth Study. Biomed Res Int 2015;1:369273.

10. Marrelli M, Tatullo M. Influence of PRF in the healing of bone and gingival tissues. Clinical and histological evaluations. Eur Rev Med Pharmacol Sci 2013;17(14):1958.

This is an open access article distributed under the terms of the Creative Commons Attribution-NonCommercial-ShareAlike 3.0 License, which allows others to remix, tweak, and build upon the work non-commercially, as long as the author is credited and the new creations are licensed under the identical terms

This article was originally published in a special issue: Special issue on "Drug Development and Human Health in China"

Indian J Pharm Sci 2020:82(1)spl issue2;21-26 